\title{
17 SUPERVISÃO DE ESTUDANTES EM ENSINO CLÍNICO: CORRELAÇÃO ENTRE DESENVOLVIMENTO DE COMPETÊNCIAS EMOCIONAIS E FUNÇÃO DE SUPORTE
}

\author{
| Paula Diogo'; Joana Rodrigues²; Odete Lemos e Sousa ${ }^{3}$; Hugo Martins'; Nuno Fernandes ${ }^{5}$ |
}

\begin{abstract}
RESUMO
Os estudantes de enfermagem têm que lidar com situações emocionalmente intensas nas interações de cuidados. Estas experiências comportam um trabalho emocional de dupla centralidade (aprendizagens ao nível da gestão das emoções dos clientes e ao nível do seu mundo emocional interno). Esta problemática conduz a um estudo com a finalidade de analisar o efeito da função de suporte do enfermeiro supervisor no desenvolvimento de competências dos estudantes de enfermagem em ensino clínico, para o desempenho do trabalho emocional. É um estudo exploratório, descritivo e correlacional, de abordagem mista. A amostra é intencional e tem como participantes 168 estudantes do $4 .^{\circ}$ ano do Curso de Licenciatura em Enfermagem e 25 enfermeiros supervisores que realizaram o Curso de Supervisão em Ensino Clínico de uma Escola Superior de Enfermagem portuguesa. Recorreu-se a um questionário aplicado aos estudantes e a jornais de aprendizagem elaborados pelos enfermeiros, como instrumentos de recolha de dados. Os dados quantitativos foram submetidos a análise estatística descritiva do tipo não-paramétrico e os dados qualitativos foram sujeitos a análise de conteúdo convencional, procedendo-se ao respetivo cruzamento. Os resultados revelam que as necessidades formativas e as expetativas dos estudantes face à supervisão são consonantes com as estratégias de suporte de gestão emocional mobilizadas pelos enfermeiros supervisores. Tendo em conta a hipótese de estudo, corrobora-se que a função de suporte do enfermeiro supervisor é potenciadora do desenvolvimento de competências para o desempenho do trabalho emocional no estudante de enfermagem em ensino clínico.
\end{abstract}

\section{PALAVRAS-CHAVE: Supervisão; Estudantes de enfermagem; Inteligência emocional; Competência clínica}

\section{RESUMEN}

"Supervisión de estudiantes de enfermería en formación clínica: Correlación el desarrollo de habilidades emocionales y la función de apoyo"

Los estudiantes de enfermería se enfrentan a situaciones emocionalmente intensas en las interacciones de cuidado. Estas experiencias implican una doble centralidad del trabajo emocional (aprendizaje en relación a la gestión de las emociones del cliente y su mundo emocional interior). Este problema lleva a un estudio con el fin de analizar el efecto de la función de apoyo de los enfermeros supervisores en el desarrollo de habilidades de los estudiantes de enfermería en la formación clínica para el desempeño del trabajo emocional. Es un estudio exploratorio, descriptivo y correlacional, de diseño mixto. La muestra es intencional siendo sus participantes 168 estudiantes del cuarto año del curso de Grado en Enfermería y 25 enfermeros supervisores con curso de supervisión en la formación clínica realizado en una escuela de enfermería portuguesa. Para la colección de dados se usaron los diarios reflexivos de los enfermeros supervisores, así como un cuestionario a los estudiantes. Los datos cuantitativos fueron sometidos a análisis estadístico descriptivo y no paramétrico; y los datos cualitativos fueron sometidos a análisis de contenido convencional, procediendo a la posterior intersección. Los resultados indican que las necesidades y expectativas de formación de los estudiantes de enfermería son consistentes con las estrategias de apoyo de gestión emocional movilizados por los enfermeros supervisores. Considerando la hipótesis de investigación, este estudio confirma que la función de apoyo del enfermero supervisor potencia el desarrollo de habilidades para el desempeño del trabajo emocional en el estudiante de enfermería en formación clínica.

\section{DESCRIPTORES: Salud mental; Educación de pregrado en medicina; Desinstitucionalización}

\begin{abstract}
"Supervision of nursing students in clinical education: Correlation between the support function and the development of emotional skills"

Nursing students deal with emotionally intense experiences in care interactions. Those experiences require a double-centred emotional labour (managing clients' emotions and their own internal emotional world). This constitutes the research problem that leads to a study aiming at analysing the support role of the clinical instructors and its correlation with the development of nursing students' emotional skills in clinical practice, required to effectively engaging in emotional labour. It is an exploratory study, with a mixed method research design, involving descriptive and correlational analysis. The sample is intentional, comprising 168 final-year students and 25 clinical instructors who attended a clinical supervision programme at a Portuguese nursing school. Data collection instruments were a questionnaire applied to the students and reflexive journals of clinical instructors. Quantitative data were submitted to descriptive, non-parametric statistical analysis whereas qualitative data were submitted to conventional content analysis; quantitative and qualitative data were crossed. Findings reveal that students' educational needs and expectations related to clinical supervision are consonant with support and emotional management strategies provided by clinical instructors. The support role of clinical instructors contributes to the development of nursing students' skills for engaging in effective emotional labour; thereby, the research hypothesis is confirmed.
\end{abstract}

\section{KEYWORDS: Supervision; Nursing students; Emotional intel- ligence; Clinical competence}

Submetido em 22-07-2016

Aceite em 10-09-2016

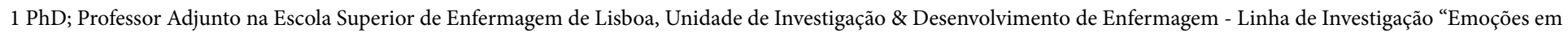
Saúde”, Av. Prof. Egas Moniz, 1600-190 Lisboa, Portugal, pmdiogo@esel.pt

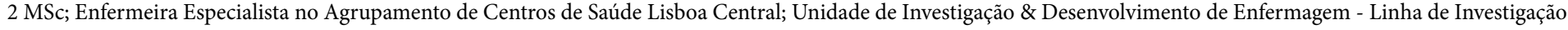
"Emoções em Saúde", 1600-190 Lisboa, Portugal, guarda.joana@gmail.com

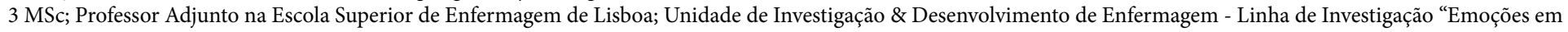
Saúde”, 1600-190 Lisboa, Portugal, olemos@esel.pt

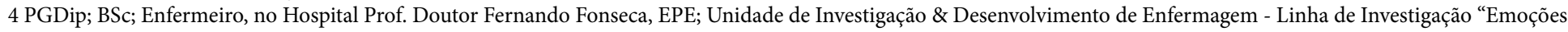
em Saúde”, 1600-190 Lisboa, Portugal, martins.hdoncalves@gmail.com

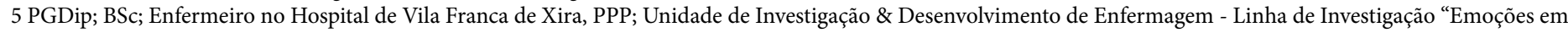
Saúde”, 1600-190 Lisboa, Portugal, fernandes.npfs@gmail.com

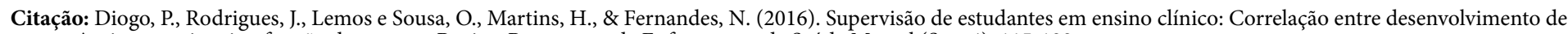
competências emocionais e função de suporte. Revista Portuguesa de Enfermagem de Saúde Mental (Spe. 4), 115-122 


\section{INTRODUÇÃO}

A supervisão clínica é uma temática essencial para enfermagem enquanto disciplina e profissão, uma vez que é determinante no percurso académico dos estudantes de enfermagem, particularmente no que respeita à componente de ensino clínico (Diogo, Rodrigues, Caeiro, e Sousa, 2016). Revela-se como um instrumento que envolve um processo focalizado de reflexão na, para e sobre a prática, objetivando a sua melhoria e a obtenção de ganhos em saúde (Falender \& Shafranske, 2014). Embora a supervisão clínica em enfermagem se centre essencialmente na supervisão de enfermeiros em exercício profissional, este conceito torna-se extensivo ao período de formação (Silva, Pires, \& Vilela, 2011), no qual o estudante contacta com diferentes contextos clínicos, o que lhe permite experienciar uma complexidade de ações/interações de cuidados nas suas várias dimensões. Esta diversidade de experiências enquadra-se nos objetivos desejados para o crescimento e aprendizagem do estudante de enfermagem. No entanto, e apesar destas experiências serem essenciais e ricas, são também conotadas com uma forte dimensão emocional, cujo impacto pode ser perturbador para o estudante devido à sua intensidade e exigência. $\mathrm{O}$ enfermeiro supervisor está, por isso, numa posição privilegiada de suporte ao implementar intervenções que visam transformar positivamente as vivências intensas e perturbadores dos clientes (e dos próprios estudantes/enfermeiros), com a intencionalidade de promover o bem-estar global das pessoas envolvidas (Diogo, 2015). A formação dos estudantes deve por isso "seguir no sentido de uma individualização do processo ensino/aprendizagem, de forma a contribuir para "o desenvolvimento total da pessoa - espírito e corpo, inteligência, sensibilidade, sentido estético, responsabilidade pessoal, espiritualidade" (Delors et al., 2010, p. 99).

De igual modo, o metaparadigma de cuidados de saúde associado à teoria de Jean Watson (2002) salienta a importância da "totalidade da natureza do indivíduo, no seu domínio físico, social, estético e moral" (Watson, 2002, p. 86), enquadrando o enfermeiro e o cliente numa dinâmica onde "estão ambos num processo de serem e de se tornarem" (Watson, 2002, p. 104), sendo esta concepção orientada pelo paradigma da transformação, segundo o qual se rege a filosofia dos cuidados de enfermagem na atualidade.
Esta atenção para com a singularidade do indivíduo, assim como para com a emocionalidade presente no processo relacional, implica o desenvolvimento simultâneo de "competências pessoais, relacionais, sociais e afetivas que "abrem a porta» às competências cognitivas e técnicas" (Rabiais, 2010, p. 45). Assim, o cerne da supervisão é procurar uma complementaridade entre aspetos emocionais e cognitivos, os quais não podem ser vistos isoladamente (Rabiais, 2010). É neste sentido que a supervisão clínica requer o desenvolvimento de competências emocionais enquanto "capacidade adquirida baseada na inteligência emocional" (Goleman, 1999 , p. 38), ou seja na capacidade de gerir os sentimentos e as emoções no plano pessoal e nas relações. O trabalho emocional (emotional labour) resulta na “indução ou supressão dos sentimentos para manter uma aparência exterior que resulte num cuidado com os sentimentos dos outros, proporcionando um ambiente seguro" (Hochschild, 1983, p. 36), pelo que assume valor de destaque neste processo. Smith (2012), pioneira no estudo sobre o trabalho emocional em enfermagem, defende que este tem uma dupla centralidade - direciona-se tanto para profissionais como para clientes dos cuidados de saúde -, isto é, os estudantes/enfermeiros devem aprender a gerir as suas próprias emoções e as dos clientes. Identifica, também, as componentes do trabalho emocional do enfermeiro como sendo: dar suporte e tranquilidade, delicadeza e amabilidade, simpatia, animar, utilizar o humor, ser agradável, ter paciência, aliviar o sofrimento, compaixão, conhecer o cliente e ajudar a resolver os seus problemas. Ainda de acordo com a mesma autora, o trabalho emocional apresenta várias características: o contacto face-a-face ou de voz com pessoas; a produção de um estado emocional na outra pessoa; a presença de um grau de controlo sobre as atividades de trabalho emocional através da formação e da supervisão clínica. O estudo de Smith (2012), sobre a aprendizagem dos estudantes de enfermagem do trabalho emocional, sugere que estas competências são, na sua maioria, aprendidas informalmente no local de trabalho, e menos em contexto de formação, pelo que os estudantes aprendem a gerir as emoções adotando uma abordagem orientada pela tarefa no seu cuidado. Por este motivo, a autora defende que o trabalho emocional é de natureza especializada e que tem de ser aprendido, da mesma forma que as competências de prestação de "cuidados físicos", dando ênfase à aprendizagem da gestão das emoções, mas também às componentes do trabalho emocional do enfermeiro. 
Destaca-se, então, que o desempenho do trabalho emocional se fundamenta na complexidade do conceito de cuidado humano (Watson, 2002) e que está presente não só no quotidiano dos enfermeiros, mas também na vertente formativa, incrementando a sua qualidade quando é mobilizado pelos enfermeiros supervisores no desempenho da sua função de suporte. O Modelo de Supervisão Interativa de Proctor (1986) enquadra a função de suporte como estando focalizada nas respostas emocionais e no suporte necessário às funções e responsabilidades inerentes às exigências do contexto, ou seja, está centrada no apoio às ações e respostas do supervisado com vista não só à diminuição do stress, mas também à sustentação das emoções adequadas às situações (Cruz, 2008), conduzindo, assim, ao desenvolvimento de competências emocionais (capacidades baseadas na gestão dos sentimentos e das emoções no plano pessoal e nas relações sociais - perceção, autorregulação, motivação, empatia e aptidões sociais), que são fundamentais para o desempenho do trabalho emocional em enfermagem. Para além do modelo de Proctor, outros modelos enquadram a função de suporte como uma das intervenções que desempenham um papel essencial na perceção dos estudantes como bem-sucedidos (Sloan \& Watson, 2001). Face ao exposto, os contributos da evidência científica permitem estabelecer conexões entre a função de suporte da supervisão (Proctor, 1986), o trabalho emocional em enfermagem (Smith, 2012), a experiência emocional de cuidar vivida pelo estudante e, consequentemente, como esta determina o desenvolvimento de competências emocionais. O desenvolvimento destas competências é fundamental para gerir emocionalmente as situações intensas da prática de cuidados de enfermagem e a função de suporte (Proctor, 1986) do enfermeiro supervisor poderá influenciar o desenvolvimento destas competências. Este estudo surge, assim, da necessidade de aprofundar a função de suporte da supervisão clínica, e de clarificar e explicitar as conexões desta função com o desenvolvimento de competências emocionais dos estudantes de enfermagem em ensino clínico, tendo sido definida a pergunta de investigação: "De que forma a função de suporte do enfermeiro supervisor é potenciadora do desenvolvimento de competências emocionais no estudante de enfermagem em ensino clínico?”. Tem como finalidade analisar o efeito da função de suporte do enfermeiro supervisor no desenvolvimento de competências dos estudantes de enfermagem em ensino clínico para o desempenho do trabalho emocional.

\section{METODOLOGIA}

Este estudo é exploratório, descritivo e correlacional, de abordagem mista. Os participantes são estudantes do $4 .^{\circ}$ ano do Curso de Licenciatura em Enfermagem (CLE) e enfermeiros orientadores de estudantes que realizaram o 3. ${ }^{\circ}$ Curso de Supervisão de Estudantes de Enfermagem em Ensino Clínico (regime b-learning). A amostra é constituída por 168 estudantes e por 25 enfermeiros supervisores de ensino clínico. Recorreu-se a uma técnica de amostragem intencional e de conveniência, pelo que as inferências indutivas dizem unicamente respeito à amostra analisada. Foi construído um questionário misto sobre a função de suporte do enfermeiro supervisor/orientador e o desenvolvimento de competências para o desempenho do trabalho emocional, na ótica dos estudantes do CLE quando estão em ensino clínico. Este é composto por três questões de resposta aberta e uma questão de resposta fechada que contempla 24 itens (grelha associada a uma escala de importância), tendo sido elaborado com base na revisão de literatura (sobre desenvolvimento de competências emocionais e função dos enfermeiros supervisores), tendo-se optado pela mobilização do Modelo de Supervisão Interativa de Proctor (1986) para a função de suporte. Os 24 itens que compõem a grelha correspondem às características da função de suporte do supervisor clínico, e são adaptados do artigo científico de Brunero e Stein-Parbury (2008). Esta grelha integra as variáveis que pretendemos medir, sob a forma de afirmações que podem ser classificadas segundo uma escala de Likert, desde "nada importante" a "muito importante" (de 1 a 5). Foi realizado o pré-teste a 8 estudantes pertencentes à amostra a inquirir. A aplicação deste instrumento permitiu obter dados quantitativos e qualitativos. A par da aplicação do questionário aos estudantes realizou-se a análise documental de jornais de aprendizagem elaborados pelos enfermeiros, segundo o modelo estruturado do Ciclo de Gibbs, que frequentaram o 3. ${ }^{\circ}$ Curso de Supervisão de Estudantes de Enfermagem em Ensino Clínico (regime b-learning). A seleção dos jornais de aprendizagem foi realizada através da leitura integral dos 243 documentos, que se encontravam depositados na plataforma informática do Curso de Supervisão, tendo sido analisadas a descrição e a reflexão sobre a função de suporte, as situações emocionalmente intensas vividas pelos estudantes que exigiram supervisão e os resultados no desenvolvimento de competências nesses estudantes. O recurso a este instrumento permitiu obter dados qualitativos. 
O tratamento de dados quantitativos foi realizado no programa SPSS ${ }^{\oplus}$ (Statistical Package for the Social Sciences), versão 20, - estatística descritiva do tipo nãoparamétrico. A partir da grelha de itens que integra o questionário elaborou-se uma base de dados para facilitar o tratamento dos mesmos. As variáveis em estudo foram os 24 itens da grelha do questionário. Os dados de natureza qualitativa foram sujeitos a análise de conteúdo convencional. Foi ainda realizado o cruzamento de dados quantitativos com dados qualitativos de modo a complementar a informação obtida e a enriquecer a análise. O pedido de autorização para recolha de dados foi realizado em novembro de 2012, à Presidência e Comissão de Ética da Escola Superior de Enfermagem. Obtivemos parecer positivo através da Presidente do Conselho Técnico-Científico, em fevereiro de 2013. A aplicação dos questionários aos estudantes realizou-se no dia 20 de fevereiro, num auditório, no final da sessão de introdução ao ensino clínico do $4 .^{\circ}$ ano (8 turmas, 288 estudantes). No sentido de salvaguardar a condição ditada pela Comissão de Ética/Presidência da Instituição - "A aplicação dos instrumentos de recolha de dados junto dos estudantes não deverá ser realizada por professores" -, dois colaboradores da linha de investigação "Emoções em Saúde" (não docentes) apresentaram aos estudantes o estudo e solicitaram a sua participação. Cada estudante, que optou por participar, respondeu individualmente ao questionário, anonimamente. A análise documental dos jornais de aprendizagem elaborados pelos enfermeiros formandos do 3. ${ }^{\circ}$ Curso de Supervisão de Estudantes de Enfermagem em Ensino Clínico (regime b-learning) foi realizada entre maio e setembro de 2013.

\section{RESULTADOS E DISCUSSÃO}

Os estudantes participantes definem trabalho emocional em enfermagem como produto, como desempenho, como trabalho stressante, e como trabalho sobre si próprio. As subcategorias obtidas relativas ao conceito de trabalho emocional, quer como produto (na medida em que permite transformar uma emoção, através de um processo, por forma a alcançar um resultado/benefício), quer como desempenho (no que diz respeito, às características necessárias para o seu exercício) vão ao encontro da definição e das características deste conceito (Hochschild, 1983; Smith, 2012), assim como das componentes do trabalho emocional do enfermeiro (Smith, 2012).
Contudo, os estudantes também definem o trabalho emocional como trabalho stressante, ou seja, enquanto efeito da atividade de cuidar sobre as suas emoções, nomeadamente, o sofrimento, as sensações dolorosas e perturbadoras. Vários autores reforçam os resultados encontrados, nomeadamente o enfoque dado às situações emocionalmente intensas (Smith, 2012; Diogo, 2015)

$\mathrm{Na}$ categoria como trabalho sobre si próprio, os estudantes referem-se ao trabalho emocional enquanto processo interno de gestão das emoções, que contempla o autoconhecimento, a compreensão do impacto das emoções e a expressão, reflexão e o controlo das emoções e sentimentos, sendo este trabalho contínuo no tempo. Este trabalho emocional exige inteligência emocional, tal como nos é apresentada por Goleman (1999) e Feather (2009), uma vez que implica a autoperceção e a autorregulação.

No que diz respeito às necessidades formativas para o desempenho do trabalho emocional, os estudantes referem as necessidades de dimensão reflexiva, emocional, cognitiva e contextual. As necessidades de dimensão reflexiva centram-se na partilha sistemática na e sobre a ação, das emoções e pensamentos, e dos conhecimentos mobilizados. São inúmeros os autores que corroboram esta necessidade de partilha dos estudantes, sublinhando o papel dos distintos parceiros neste processo (Alarcão e Rua, 2005). Smith e Gray (2001) referem que o trabalho emocional é cumulativo através da experiência de enfermagem, e provém da capacidade de refletir sobre essa experiência com a equipa e colegas. Também Benner (2001) defende que os jornais de aprendizagem são instrumentos fundamentais para a análise das práticas, permitindo o desenvolvimento da reflexão na e sobre a ação. Quanto à dimensão emocional, os estudantes identificaram necessidades formativas no âmbito da gestão emocional, no plano intrapessoal e no plano interpessoal. Algumas das necessidades formativas integram as componentes do modelo apresentado por Mayer e Salovey (1997), no qual estão descritas as quatro dimensões que compõem a Inteligência Emocional, nomeadamente: (1) a perceção, avaliação e expressão da emoção; (2) a integração e assimilação da emoção; (3) a compreensão e análise de emoções aplicando conhecimento emocional; (4) a regulação reflexiva das emoções que promove o crescimento emocional e intelectual. No plano intrapessoal os estudantes referem ter necessidade de formação promotora do autoconhecimento, o que vai ao encontro do defendido por Rabiais (2010). 
A dimensão cognitiva também foi valorizada pelos estudantes, nomeadamente no que diz respeito às técnicas e componentes do trabalho emocional, ao processo emocional, à relação interpessoal, assim como à pessoa enquanto sistema, aos fenómenos de saúde-doença e às intervenções de suporte. Através destes resultados verifica-se uma complementaridade entre aspetos emocionais e cognitivos que, tal como refere Rabiais (2010), não podem ser encarados isoladamente, mas sim com um estreito grau de interdependência. Transversal a estas dimensões surge o contexto das práticas, traduzido na dimensão contextual, que os estudantes incluem como necessidade formativa. Nesta linha de pensamento, Rogers (1969) destacava a importância do ambiente onde a aprendizagem ocorre, devendo o mesmo fornecer uma configuração de confiança e de apoio no qual o estudante se sinta seguro para explorar os seus sentimentos e expressar as suas opiniões. Integrados nesta categoria surgem os atributos que os estudantes valorizam no enfermeiro supervisor para o desempenho do trabalho emocional, nomeadamente, o humanismo, a visão holística, a capacidade para promover a expressão de sentimentos, a interação afetivo-emocional e a gestão de emoções. São vários os autores que enunciam os atributos referidos (Smith \& Gray, 2001; Rabiais, 2010). A função de suporte, tal como é apresentada por Proctor (1986), coaduna-se com estes resultados, uma vez que realça que o processo supervisivo se concretiza no apoio às ações e respostas do estudante, de modo a que o mesmo supere situações significativas, não só diminuindo o stress que vivencia como adequando as emoções às situações.

No que diz respeito às expetativas face à orientação do supervisor clínico para o desempenho do trabalho emocional, os estudantes valorizam que este promova o trabalho sobre si próprio, ou seja, esperam que o supervisor clínico os conduza ao autoconhecimento, através da promoção da reflexão sobre as emoções vividas, fomentando a descentração do próprio Eu, o que está em consonância com as necessidades formativas (Abreu, 2002; Smith \& Gray, 2001). Outra expetativa mencionada diz respeito à promoção de competências facilitadoras do estabelecimento de relações interpessoais, ou seja, da gestão interna impulsionadora do estabelecimento de uma relação com o Outro, seja com a equipa, com os colegas ou com os clientes. Neste âmbito, foi destacado o papel do enfermeiro supervisor na promoção da interação com o cliente, em que o enfermeiro sugere estratégias ao estudante para o ajudar a lidar com as situações, e lhe proporciona espaço para a interação.
Rabiais (2010) reforça que frequentemente as necessidades emocionais sentidas pelos estudantes de enfermagem prendem-se com o facto das relações interpessoais não serem as mais adequadas. A inteligência emocional e o trabalho das emoções são apontados por vários autores como elemento fulcral do desenvolvimento desta competência (Freshwater \& Stickley, 2004). Os estudantes esperam do supervisor, ainda, a capacidade de promover a gestão emocional das situações. Quanto aos supervisores clínicos e às características facilitadoras da aprendizagem do trabalho emocional valorizadas pelos estudantes, estas estão alinhadas com as apresentadas por Brunero e Stein-Parbury (2008), relativas à função de suporte do enfermeiro supervisor. Interessante também é verificar que as características que emergiram nesta subcategoria estão relacionadas com os atributos do enfermeiro supervisor valorizados na dimensão contextual, inerente às necessidades formativas dos estudantes. A promoção de um ambiente de suporte foi valorizado pelos estudantes como facilitador do desempenho do trabalho emocional.

Por seu lado, a análise das características da função de suporte do supervisor clínico quantificadas pelos estudantes permite identificar aquelas que se configuram como principais meios de aprendizagem do desempenho do trabalho emocional em enfermagem. Dos resultados relativos à questão fechada do questionário aplicado aos estudantes destacam-se as características da função de suporte que obtiveram uma classificação de 4 ou 5 ("muito importante") superior a 90\%: escutar e compreender (96.4\%), promover a autoconfiança (95.8\%), promover o sentimento de segurança no grupo (95.2\%), promover a capacidade de gerir emocionalmente as situações (94.6\%), garantir o bom relacionamento com a equipa (92.9\%), manter o envolvimento com o contexto de cuidados $(92.9 \%)$, incrementar o interesse pela experiência (91.1\%), evitar o tédio e a ausência de interesses (91.0\%), aliviar e atenuar a experiência (90.4\%), construir uma estratégia de coping enquanto recurso e disponibilidade para ajudar a lidar com as situações (90.4\%), favorecer o relacionamento com os enfermeiros (90.4\%).

O cruzamento dos dados quantitativos com os dados qualitativos obtidos através do questionário aplicado aos estudantes permite, por um lado, compreender a importância atribuída à função de suporte do enfermeiro supervisor, e por outro identificar as características de suporte mais valorizadas pelos estudantes. 
Da análise aos jornais de aprendizagem elaborados pelos enfermeiros emerge uma compreensão da perspetiva dos supervisores clínicos em relação à sua função de suporte na orientação de estudantes, salientando-se os seus pontos de vista e pressupostos que constituem antecedentes fundamentais para o desempenho da função de supervisão (Proctor, 1986). As perspetivas emergentes sobre o processo supervisivo e a função de suporte focalizam a valorização global da supervisão, as experiências/respostas emocionais dos estudantes e o suporte necessário às funções e responsabilidades inerentes às exigências do contexto, ou seja, centradas no apoio às ações e respostas do supervisado com vista não só à diminuição do stress como também à sustentação das emoções adequadas às situações (Cruz, 2008).

Os resultados revelam ainda que no contexto clínico o estudante de enfermagem vivencia de forma muito intensa os problemas das pessoas que cuida, absorvendo as respostas emocionais de tal sofrimento e revelando sinais de stress e disrupção emocional (Morse, Bottorff, Anderson, O’Brien, \& Solberg, 2006). As situações emocionalmente intensas identificadas pelos enfermeiros participantes são: o período de integração, o sofrimento e morte do cliente, a construção do relacionamento com o cliente, a realização de procedimentos, o perfil/estado emocional do estudante, os conflitos e o momento de avaliação. Os enfermeiros participantes consideram que a supervisão e a função de suporte envolvem tanto a gestão das emoções dos estudantes como a gestão das emoções dos clientes no processo de cuidados (Smith, 2012), mas este suporte tem como alicerce fundamental a relação estabelecida entre enfermeiro supervisor e estudante, e visa o desenvolvimento de competências emocionais nos estudantes. A análise de conteúdo aos jornais de aprendizagem dos enfermeiros fez ainda emergir categorias que caracterizam o seu desempenho da função de suporte na supervisão de estudantes em ensino clínico (cinco categorias), que foram agrupadas em dois tipos:

- Tipo I - Abordagem direta na gestão emocional - os enfermeiros procuram fomentar a gestão emocional dos estudantes e da relação de cuidados ao processar o estado emocional (promover a autoconfiança e autoestima, transmitir tranquilidade, motivar) e ao elaborar as emoções (compreender as vivências emocionais, facilitar a gestão emocional).

- Tipo II - Abordagem indireta na gestão emocional - os enfermeiros procuram construir a relação enfermeiro supervisor/estudante, fornecer orientações sobre dinâmica do serviço e dos cuidados, refletir sobre as práticas e ensino clínico e ainda mobilizar a modelagem.
As características da função de suporte do supervisor clínico (Brunero \& Stein-Parbury, 2008) corroboram em muitos aspetos as estratégias emergentes, que se enquadram na perspetiva holística e construtivista da formação, e que contribuem para o desenvolvimento de competências emocionais. Estas competências são fulcrais no trabalho emocional inscrito no cuidar humano e holístico descrito por Diogo (2015): (1) promover um ambiente seguro e afetuoso; (2) nutrir os cuidados com afeto; (3) gerir as emoções dos clientes, (4) construir a estabilidade na relação; (5) regular a disposição emocional para cuidar.

As necessidades e estratégias formativas para o desempenho do trabalho emocional identificadas pelos estudantes coincidem, em grande parte, com estratégias realçadas pelos enfermeiros supervisores como potenciadoras do desenvolvimento de competências emocionais, o que é corroborado igualmente pela evidência científica. Destaca-se das estratégias formativas para o desempenho do trabalho emocional: análise das práticas no local de trabalho com enfermeiros experientes, treino de competências emocionais centradas no cliente, treino centrado no autoconhecimento, aprendizagem reflexiva/supervisão clínica; técnicas de dinâmica de grupo, reflexão sobre a prática, autoconhecimento das emoções; treino de autoconhecimento, sessões de grupo para análise de situações/supervisão clínica; prática reflexiva. Os resultados evidenciam, ainda, a importância do clima de confiança, ambiente de abertura e tranquilidade que favorece a reflexão sobre as práticas e a expressão emocional na relação enfermeiro supervisor/estudante.

O desempenho da função de suporte, tendo em conta as diversas estratégias mobilizadas no processo de supervisão, tem repercussões no desenvolvimento de competências no estudante (Quadro 1).

Os resultados em termos de desenvolvimento de competências nos estudantes demonstram que a função de suporte do enfermeiro supervisor potencia o desempenho do trabalho emocional e promove o crescimento pessoal, conduzindo a mudanças de atitude (postura, discurso, análise, adaptação e tranquilidade), ao desenvolvimento de confiança, iniciativa e autonomia, ao reconhecimento das dificuldades e à aceitação da crítica de modo construtivo. Mas também conduz à aquisição de conhecimentos teóricos resultantes da confrontação com outras formas de interpretação das circunstâncias, exigindo uma adaptação a novas experiências e a um domínio de termos científicos. 
Quadro 1 - Promoção do Desenvolvimento e de Competências Emocionais no Estudante de Enfermagem

\begin{tabular}{|c|c|}
\hline Subcategorias & Categorias \\
\hline $\begin{array}{l}\text { Mudanças de atitude: postura, discurso, análise, } \\
\text { adaptação, tranquilidade }\end{array}$ & \multirow{4}{*}{ Crescimento pessoal } \\
\hline Desenvolver a confiança & \\
\hline Desenvolver a iniciativa e autonomia & \\
\hline $\begin{array}{l}\text { Reconhecer as dificuldades e aceitar a crítica de } \\
\text { modo construtivo }\end{array}$ & \\
\hline $\begin{array}{l}\text { Adquirir de novos conhecimentos na confrontação } \\
\text { com outra formas de interpretação das circunstân- } \\
\text { cias e com a adaptação a novas experiências }\end{array}$ & \multirow[t]{2}{*}{$\begin{array}{l}\text { Aquisição de conheci- } \\
\text { mentos teóricos }\end{array}$} \\
\hline Domínio de termos científicos & \\
\hline Aumentar a confiança & \multirow{4}{*}{$\begin{array}{l}\text { Aperfeiçoar as habili- } \\
\text { dades relacionais }\end{array}$} \\
\hline Trabalhar a autoestima & \\
\hline $\begin{array}{l}\text { Consciencializar do fluxo de emoções na relação de } \\
\text { cuidados }\end{array}$ & \\
\hline $\begin{array}{l}\text { Compreender o potencial terapêutico da relação de } \\
\text { cuidados }\end{array}$ & \\
\hline Aperfeiçoar o desempenho & \multirow{3}{*}{$\begin{array}{l}\text { Otimizar o desem- } \\
\text { penho profissional }\end{array}$} \\
\hline $\begin{array}{l}\text { Compreender os resultados terapêuticos das ações } \\
\text { de enfermagem }\end{array}$ & \\
\hline $\begin{array}{l}\text { Evitar que o estado emocional do estudante con- } \\
\text { tamine a relação de cuidados }\end{array}$ & \\
\hline Compreender as suas emoções e sentimentos & \multirow{5}{*}{$\begin{array}{c}\text { Mobilizar estratégias } \\
\text { de gestão emocional } \\
\text { centradas no cliente e } \\
\text { no próprio }\end{array}$} \\
\hline $\begin{array}{l}\text { Libertar e descomprimir através da expressão } \\
\text { emocional }\end{array}$ & \\
\hline Ajudar o cliente a sentir calma e serenidade & \\
\hline $\begin{array}{l}\text { Aprender a gerir situações de stress influenciando } \\
\text { positivamente o desempenho }\end{array}$ & \\
\hline Manter a estabilidade emocional & \\
\hline
\end{tabular}

A função de suporte permite, ainda, aperfeiçoar as habilidades relacionais, aumentando a confiança, trabalhando a autoestima, consciencializando o estudante para o fluxo de emoções na relação de cuidados e ajudando-o a compreender o potencial terapêutico da relação de cuidados. Tudo isto permite otimizar o desempenho profissional, pois o estudante gradualmente consegue compreender os resultados terapêuticos das ações de enfermagem e evitar que o seu estado emocional contamine a relação de cuidados, passando a mobilizar estratégias de gestão emocional centradas no cliente e no próprio. Desta forma, o estudante consegue: compreender as suas emoções e sentimentos; libertar e descomprimir (através da expressão emocional); ajudar o cliente a sentir calma e serenidade; aprender a gerir situações de stress, influenciando positivamente o desempenho e regulando a sua estabilidade emocional. Sem um envolvimento emocional qualquer ação, ideia ou decisão assentaria exclusivamente em bases racionais, pelo que Damásio (2012) defende que o desenvolvimento emocional faz parte integrante do processo de tomada de decisões e funciona como um vetor de ações e ideias, consolidando a reflexão e o discernimento.
Assim sendo, a formação em enfermagem "não se deve confinar ao processo técnico e científico, mas também sublinhar o desenvolvimento pessoal do estudante pelo papel gerador que tem em todas as competências" (Rabiais 2010, p. 36), ou seja, não é possível separar as dimensões cognitivas, sociais, afetivas e emocionais quando se pretende clarificar os fatores que estão na base do desenvolvimento da aprendizagem do Cuidar de Enfermagem. Também as emoções são, então, uma dimensão essencial não só para o enfermeiro mas também para o estudante, que procura o sucesso muitas vezes num emaranhado turbulento de experiências, pensamentos e emoções que precisa de aprender a gerir.

\section{CONCLUSÕES E IMPLICAÇÕES PARA A PRÁTI- CA CLÍNICA}

Os estudantes demonstram conhecer a conceção de trabalho emocional, o que revela que este é trabalhado pelo menos de uma forma implícita no Plano de Estudos da Licenciatura de Enfermagem. Além disso, identificam necessidades formativas de dimensão reflexiva, emocional, cognitiva e contextual para o desempenho do trabalho emocional. As características da função de suporte do supervisor clínico, bem como os resultados do questionário aos estudantes, corroboram em diversos aspetos as estratégias identificadas pelos enfermeiros supervisores, revelando um conjunto de estratégias diretas (Tipo I) e indiretas (Tipo II) de gestão emocional, que contribuem para o desenvolvimento de competências para o trabalho emocional em enfermagem. Também as respostas dos estudantes são consonantes, em termos das necessidades formativas e expetativas face à supervisão, com as estratégias de suporte mobilizadas pelos enfermeiros supervisores que se encontram a frequentar o curso de supervisão.

Destaca-se, ainda, que o ambiente de suporte é essencial na supervisão dos estudantes, visando a partilha, confiança e abertura para a expressão e gestão emocional. Nesta supervisão de suporte, o processo reflexivo, a descentração e o autoconhecimento são fundamentais. Os resultados deste estudo permitem apresentar uma compreensão mais aprofundada das estratégias de supervisão, mobilizadas pelos enfermeiros na orientação de estudantes em ensino clínico, no âmbito da função de suporte, e ainda sobre a relação entre a supervisão, o desenvolvimento de competências emocionais e o desempenho do trabalho emocional. O desenvolvimento de competências emocionais revela-se fulcral na capacidade de gerir emocionalmente as situações intensas presentes no exercício da enfermagem, com repercussões na qualidade dos cuidados e na equipa de saúde, 
e a função de suporte do enfermeiro supervisor influencia o desenvolvimento de tais competências. Os supervisores assumem um papel central na aprendizagem dos estudantes de enfermagem, nomeadamente no envolvimento no trabalho emocional e no seu desenvolvimento enquanto enfermeiros. Estas conclusões permitem alargar a compreensão da conceção de trabalho emocional em enfermagem, e contribuir para a fundamentação da sua integração nos programas curriculares dos cursos de enfermagem desde a formação inicial com impacto futuro na prática de cuidados. Em estudos futuros, importa clarificar em que medida o docente é promotor do trabalho emocional no estudante de enfermagem em ensino clínico e em que medida a parceria de cuidados que estabelece com o enfermeiro supervisor potencia o desenvolvimento do trabalho emocional. Importa também compreender se o trabalho emocional promove o bemestar no cliente, ou seja, quais os resultados em saúde.

\section{REFERÊNCIAS BIBLIOGRÁFICAS}

Abreu, W. (2002). Supervisão clínica em enfermagem: Pensar as práticas, gerir a formação e promover a qualidade. Revista Sinais Vitais, 45(11), 53-57.

Alarcão, I., e Rua, M. (2005). Interdisciplinaridade, estágios clínicos e desenvolvimento de competências. Texto \& Contexto Enfermagem, 14(3), 373-382.

Benner, P. (2001). De iniciado a perito: Excelência e poder na prática clínica de enfermagem. Coimbra: Quarteto Editora.

Brunero, S., \& Stein-Parbury, J. (2008). The effectiveness of clinical supervision in nursing: An evidenced based literature review. Australian Journal of Advanced Nursing, 25(3), 86-94.

Cruz, S. (2008). A supervisão clínica em enfermagem como estratégia de qualidade no contexto da enfermagem avançada. Servir, 56(5-6), 200-206.

Damásio, A. (2012). As competências emocionais na educação.

Delors, J., Al Mufti, I., Amagi, I., Carneiro, R., Chung, F., Geremek, B., ...Nanzhao, Z. (2010). Educação: Um tesouro a descobrir. Relatório para a UNESCO da Comissão Internacional sobre Educação para o século XXI. Brasília: UNESCO.

Diogo, P. (2015). Trabalho com as emoções em enfermagem pediátrica. Um processo de metamorfose da experiência emocional no ato de cuidar ( $2^{\mathrm{a}} \mathrm{ed}$.). Loures: Lusodidacta.

Diogo, P., Rodrigues, J., Caeiro, M. J., e Sousa, O. L. (2016). The support role in clinical supervision of nursing students: Determinant in the development of emotional skills. Academia Journal of Educational Research, 4(5), 75-82.
Falender, C. A., \& Shafranske, E. P. (2014). Clinical supervision: State of the art. Journal of Clinical Psychology In Session, 70(11), 130-141.

Feather, R. (2009). Emotional intelligence in relation to nursing leadership: Does it matter? Journal of Nursing Management, 17, 376-382.

Freshwater, D., \& Stickley, T. (2004). The heart of the art: Emotional intelligence in nurse education. Nursing Inquiry, 11(2), 91-98.

Goleman, D. (1999). Trabalhando com a inteligência emocional. Rio de Janeiro: Objetiva.

Hochschild, A. (1983). The managed hearth. Commercialization of human feeling. Berkeley: University of California Press, Ltd.

Mayer, J., \& Salovey, P. (1997). What is emotional intelligence? In P. Salovey, \& D. Sluyter, Emotional development and emotional intelligence: Educational implications (pp. 3-31). New York: BasicBooks.

Morse, J. M., Bottorff, J., Anderson, G., O’Brien, B., \& Solberg, S. (2006). Beyond empathy: Expanding expressions of caring. Journal of Advanced Nursing Compliation (30th Anniversary Issue), 75-87.

Proctor, B. (1986). Supervision: A co-operative exercise in accountability. In M. Marken, \& M. Payne (Eds.), Enabling and ensuring national youth bureau and council for education and training in youth and community work. Leicester.

Rabiais, I. (2010). Sensibilidade emocional dos estudantes de enfermagem na aprendizagem de cuidar. In A. Fernandes, Â. Santos, C. Barros, E. Guilherme, M. J. Martins, M. J. Miranda, ...R. Vilela, Emoções em saúde - Contributos (pp. 36-53). Fátima: Corrente Dinâmica.

Rogers, C. (1969). Freedom to Learn: A View of What Education Might Become. (1st ed.) Columbus, Ohio: Charles Merill. Excerpts.

Silva, R., Pires, R., e Vilela, C. (2011). Supervisão de estudantes de enfermagem em ensino clínico - revisão sistemática da literatura. Revista de Enfermagem Referência, III Série(3), 113-122.

Sloan, G., \& Watson, H. (2001). John Heron's six-category intervention analysis: Towards understanding interpersonal relations and progressing the delivery of clinical supervision for mental health nursing in the United Kingdom. Journal of Advanced Nursing, 36(2), 206-214.

Smith, P., \& Gray, B. (2001). Reassessing the concept of emotional labour in student nurse education: Role of link lecturers and mentors in a time of change. Nurse Education Today, 21, 230-237.

Smith,P.(2012). Theemotionallabour of nursingrevisited:Can nurses still care? (2nd ed.). Hampshire: Palgrave Macmillan

Watson, J. (2002). Enfermagem: Ciência humana e cuidar, uma teoria de enfermagem. Loures: Lusociência. 